\title{
Proyectos de vinculación con la sociedad en las universidades ecuatorianas. Caso:
} Universidad Estatal de Milagro-Ecuador

\section{Projects of linkage with society in Ecuadorian universities. Case: State University of Milagro-Ecuador}

DOI: $10.46932 / \mathrm{sfjdv2n5-073}$

Received in: Oct 1st, 2021

Accepted in: Dec 30th, 2021

\author{
Alexandra Cecilia Astudillo Cobos \\ Docente Titular de Facultad Ciencias de la Educación \\ Universidad Estatal de Milagro \\ Milagro, Guayas, Ecuador \\ E-mail: aastudilloc@unemi.edu.ec \\ Mirella Azucena Correa Peralta \\ Docente Titular de Facultad Ciencias e Ingenierías \\ Universidad Estatal de Milagro \\ Milagro, Guayas, Ecuador \\ E-mail: mcorreap@unemi.edu.ec \\ Verónica Patricia Santos Yánez \\ Docente Titular de Facultad de Salud y Servicios Sociales \\ Universidad Estatal de Milagro \\ Milagro, Guayas, Ecuador \\ E-mail: vsantosy@unemi.edu.ec \\ Jorge Luis Vinueza Martínez \\ Docente Titular de Facultad Ciencias e Ingenierías \\ Universidad Estatal de Milagro \\ Milagro, Guayas, Ecuador \\ E-mail: jvinuezam@unemi.edu.ec
}

\section{RESUMEN}

Los proyectos de vinculación con la sociedad en las universidades se desarrollan en un marco de investigación-acción-participación, aplicando competencias teórico-prácticas, desde la sinergia de estudiantes, profesores y la comunidad cuyos resultados finales se evidencian con el informe de las actividades y resultados alcanzados. Un sistema de seguimiento y evaluación es un componente de mecanismo para: 1. Fundamentar la ejecución del programa o proyecto que fortalezca el aprendizaje institucional, 2. Difusión de conocimientos velando por la rendición de cuentas y en consonancia con las normas establecidas; y 3. Brindar oportunidades a los beneficiarios poniendo énfasis en los logros y resultados. Para el proceso de creación y ejecución de los proyectos de vinculación se definió 4 fases: 1 . Diagnóstico o línea base (análisis de situación, análisis de beneficiario, análisis de problemas), 2. Formulación del proyecto (antecedentes y justificación, objetivos del proyecto, beneficiarios, resultados, actividades, indicadores, recursos), 3. Ejecución y seguimiento, y 4. Evaluación final, que aportó significativamente con la pertinencia a la zona 5 en el Ecuador. 
Palabras Claves: Vinculación, proyecto, pertinência.

\begin{abstract}
The projects of linkage with society in universities are developed in a framework of research-actionparticipation, applying theoretical-practical skills, from the synergy of students, teachers and the community whose final results are evidenced with the report of the activities and results achieved. A monitoring and evaluation system is a component of a mechanism to: 1 . substantiate the implementation of the program or project that strengthens institutional learning, 2. disseminate knowledge ensuring accountability and in line with established standards; and 3. provide opportunities to beneficiaries with emphasis on achievements and results. For the process of creation and execution of the linkage projects, 4 phases were defined: 1. Diagnosis or baseline (situation analysis, beneficiary analysis, problem analysis), 2. Formulation of the project (background and justification, project objectives, beneficiaries, results, activities, indicators, resources), 3.
\end{abstract}

Keywords: Linkage, project, relevance.

\title{
1 PROYECTOS DE VINCULACIÓN CON LA SOCIEDAD EN LAS UNIVERSIDADES
}

La vinculación es:

\begin{abstract}
“...el conjunto de actividades universitarias relacionadas con: a) la generación de conocimiento y capacidades en colaboración con organizaciones y agentes no académicas, así como b) el uso, aplicación y explotación del conocimiento y otras capacidades existentes en la universidad, fuera del entorno académico. O más brevemente, el conjunto de interacciones entre la universidad y el resto de la sociedad” (D`Este, P.; Martínez, E. y Molas-Gallart, J. ,2009).
\end{abstract}

Pues la proyección de la vinculación con la sociedad no se refiere únicamente a un trabajo entre estudiantes y profesores en las universidades, se trata de vincular a toda la población, tanto la comunidad interna como externa. Además, la Propuesta de Plan Nacional de Vinculación de la Educación con la Sociedad se menciona al proceso de Vinculación con la sociedad como resultado de las relaciones sociales que se dan entre los sujetos que en él participan (diagnóstico), está dirigido de un modo sistémico y eficiente a la promoción de cultura y desarrollo para la comunidad (objetivo), para coadyuvar a la solución de la problemática social (problema) en la necesidad de contribuir al desarrollo de la comunidad, mediante la apropiación de la cultura que ha acumulado la sociedad en su desarrollo (contenido); a través de la participación activa de la comunidad (método); planificada en el tiempo y observando ciertas estructuras organizativas (formas); con ayuda de ciertos objetos (medios) e instrumentando indicadores que le permitan medir la calidad (evaluación)".

Pues, estos proyectos se desarrollan en un marco de investigación- acción participación, en el cual los estudiantes aplican conocimientos teóricos - prácticos relacionados a su formación universitaria, siendo los proyectos el conjunto de actividades concretas, interrelacionadas y coordinadas entre sí que se 
realizan para resolver problemas y satisfacer necesidades. La ejecución de cualquier Proyecto supone una serie de actividades secuenciadas, que apuntan al logro de un objetivo común.

Dentro del plan de vinculación con la sociedad se enmarcan pasantías, prácticas pre profesionales de los estudiantes y cursos de educación continua guiados por el personal académico, Art. 125 y 27 de la Ley Orgánica de Educación Superior (LOES), siempre y cuando estén en el planificación general. Pues, no existe "Vinculación con la Sociedad" sin que exista una planificación integral, cuyo propósito general siempre debe ser "Dejar Huellas" en un sector con la finalidad que la propia comunidad adquiera acciones para continuar con sus procesos de forma autónoma; esto no significa que la Institución de Educación Superior (IES) tenga que remitir un "Plan de Vinculación” pero si debe inferir su existencia.

Según Ander-Egg (2007), define un plan: “es el parámetro técnico-político dentro del cual se enmarcan los programas y proyectos" (p.63). Por lo tanto, según el mismo autor el Plan engloba programas y proyectos, constituyendo éste un elemento emergente, producto de la interacción entre sus partes.

Para Torres Hernández, Z., \& Torres Martínez, H. (2014) define los programas en sentido amplio, como:

"Un conjunto organizado, coherente e integrado de actividades, servicios o procesos, expresados en un conjunto de proyectos relacionados o coordinados entre sí y que son de similar naturaleza"

Además, plantea que "la diferencia entre programa y proyecto radica en la magnitud, diversidad y especificidad, habida cuenta que un programa está constituido por una constelación o conjunto de proyectos".

Como IES la Universidad Estatal de Milagro (UNEMI) asume el desafío compartido con el Reglamento de Régimen Académico (RRA), recogido en el artículo 107 de la LOES,

“...el principio de pertinencia consiste en que la educación superior responda a las expectativas y necesidades de la sociedad, a la planificación nacional, y al régimen de desarrollo, a la prospectiva de desarrollo científico, humanístico y tecnológico mundial, y a la diversidad cultural. (...)”.

Es decir, reforzar su pertinencia frente a los problemas sociales, productivos y culturales de su entorno basada en los llamados dominios científicos, tecnológicos y humanísticos de las IES ecuatorianas.

La UNEMI considera vinculación con la sociedad como una misión permanente, centrada en la Región 5, aunque con proyección a la región y el país (esto último en función a la expansión de la educación en línea). Los proyectos de vinculación benefician a la sociedad, estudiantes universitarios y docentes; pues los estudiantes entran en contacto con las necesidades reales del mundo laboral desde la perspectiva de la formación universitaria y en el caso de los docentes tienen la oportunidad desde el conocimiento responder a las demandas de las comunidades. 
Así, el plan de vinculación con la Sociedad-UNEMI 2014-2017 nació como una necesidad de un cambio de paradigma del conocimiento y del aprendizaje, que evoluciona hacia la participación de la sociedad en las redes académicas y contribuyen al desarrollo de la democracia.

Los programas y proyectos de vinculación basaron sus cimientos en los objetivos del Plan Nacional del Buen Vivir, en este caso la UNEMI, priorizó los objetivos 4, 7 y 10, con sus respectivas políticas (Tabla 01).

Tabla 01. Programas y Proyectos de Vinculación en sustentación con los objetivos del Plan Nacional del Buen vivir.

Plan Nacional del Buen Vivir

\begin{tabular}{|c|c|}
\hline Objetivo & Política \\
\hline $\begin{array}{l}\text { 4. Fortalecer las } \\
\text { capacidades } \\
\text { potencialidades de la } \\
\text { ciudadanía }\end{array}$ & $\begin{array}{l}\text { 4.9 Impulsar la formación en } \\
\text { áreas de conocimiento no } \\
\text { tradicionales que aportan a la } \\
\text { construcción del Buen Vivir }\end{array}$ \\
\hline $\begin{array}{lr}\text { 7. Garantizar } & \text { los } \\
\text { derechos de } & \text { la } \\
\text { naturaleza } & \text { y } \\
\text { promover } & \text { la } \\
\text { sostenibilidad } \\
\text { ambiental territorial y } \\
\text { global. }\end{array}$ & $\begin{array}{l}\text { 7.4 Impulsar la generación de } \\
\text { conocimiento como } \\
\text { alternativa a la producción } \\
\text { primario exportadora. } \\
\text { 7. } 7 \text { Promover la eficiencia y } \\
\text { una mayor participación de } \\
\text { energías renovables } \\
\text { sostenibles como medida de } \\
\text { prevención de la } \\
\text { contaminación ambiental }\end{array}$ \\
\hline $\begin{array}{l}\text { 10. Impulsar la } \\
\text { transformación de la } \\
\text { matriz productiva }\end{array}$ & $\begin{array}{l}10.1 \text { Diversificar y generar } \\
\text { mayor valor agregado en la } \\
\text { producción nacional. }\end{array}$ \\
\hline
\end{tabular}

Fuente: Plan Nacional del Buen Vivir

Para el periodo junio 2014-febrero 2017, se propuso seguir directrices del Modelo de evaluación del Consejo de Evaluación Acreditación y Aseguramiento de la Calidad de la Educación Superior (CEAACES) y el Consejo de Educación Superior (CES), regidos por los artículos:

Art. 125 de la Ley Orgánica de Educación Superior, contempla: "Programas y cursos de vinculación con la sociedad. - Las instituciones del Sistema de Educación Superior realizarán programas y cursos de vinculación con la sociedad guiados por el personal académico. Para ser estudiante de los mismos no hará falta cumplir los requisitos del estudiante regular” (p. 72).

En este contexto, la profesionalización docente desde la observación y reflexión permiten crear una acción crítica y evaluada en los resultados desde el quehacer académico como es la calidad de la práctica docente por medio de experiencias al ser adquiridas desde la vinculación.

Según el artículo 78 del Reglamento de Régimen Académico:

"Fortalezas o dominios académicos de las instituciones de educación superior. - Un dominio académico consiste en las fortalezas científicas, tecnológicas, humanísticas y artísticas demostradas por una IES, con base en su trayectoria académica e investigativa, personal académico 
altamente calificado, infraestructura científica y gestión pertinente del conocimiento. Las IES formularán su planificación institucional considerando los dominios académicos, los cuales podrán ser de carácter disciplinar e interdisciplinar. La referida planificación deberá ser informada a la sociedad" (CES, 2013).

En el Art. 88 del Reglamento de Régimen Académico determina que:

\begin{abstract}
"Prácticas-pre profesionales. - Son actividades de aprendizaje orientadas a la aplicación de conocimientos y al desarrollo de destrezas y habilidades específicas que un estudiante debe adquirir para un adecuado desempeño en su futura profesión. Estas prácticas deberán ser de investigación- acción y se realizarán en el entorno institucional, empresarial o comunitario, público o privado, adecuado para el fortalecimiento del aprendizaje. (...) El contenido, desarrollo y cumplimiento de las prácticas pre-profesionales serán registrados en el portafolio académico" (p.80).
\end{abstract}

En este sentido el profesional al terminar su carrera las prácticas y la relación con la colectividad por medio de la vinculación, aportará a las realidades y las necesidades de su contexto.

\title{
2 PROCESO DE PLANIFICACIÓN, EJECUCIÓN, MONITOREO Y EVALUACIÓN DE LOS PROYECTOS
}

Un sistema de seguimiento y evaluación funcional es un componente indispensable de un buen mecanismo de gestión y rendición de cuentas de proyectos y programas que permiten:

- Fundamentar la ejecución del programa o proyecto con informes que evidencien resultados que permita proporcionar a los responsables de la gestión adoptar decisiones que dirijan en la intervención y mejora de los resultados.

- Contribuir al aprendizaje institucional y a la difusión de conocimientos al compartir y reflexionar sobre las experiencias y enseñanzas.

- Velar por la rendición de cuentas y cumplimiento al demostrar si hemos llevado a cabo nuestra tarea conforme a lo acordado en consonancia con las normas establecidas.

- Brindar oportunidades para que las partes interesadas, en particular los beneficiarios, expresen sus opiniones y percepciones de la labor, mostrando como ejemplos de apertura a las críticas y exteriorizando nuestro deseo de aprender de las experiencias y de adaptarnos a las necesidades cambiantes.

- Fomentar y encomiar nuestra labor poniendo énfasis en nuestros logros y resultados, reforzando la moral y contribuyendo a la movilización de recursos. 


\section{FASES DE LOS PROYECTOS DE VINCULACIÓN}

Los proyectos es el conjunto de actividades interrelacionadas y coordinadas para la resolución de problemas para satisfacer las necesidades que apuntan al logro de un objetivo común, para ello se establece fases.

Figura 01. Fases del proceso de los proyectos de vinculación UNEMI
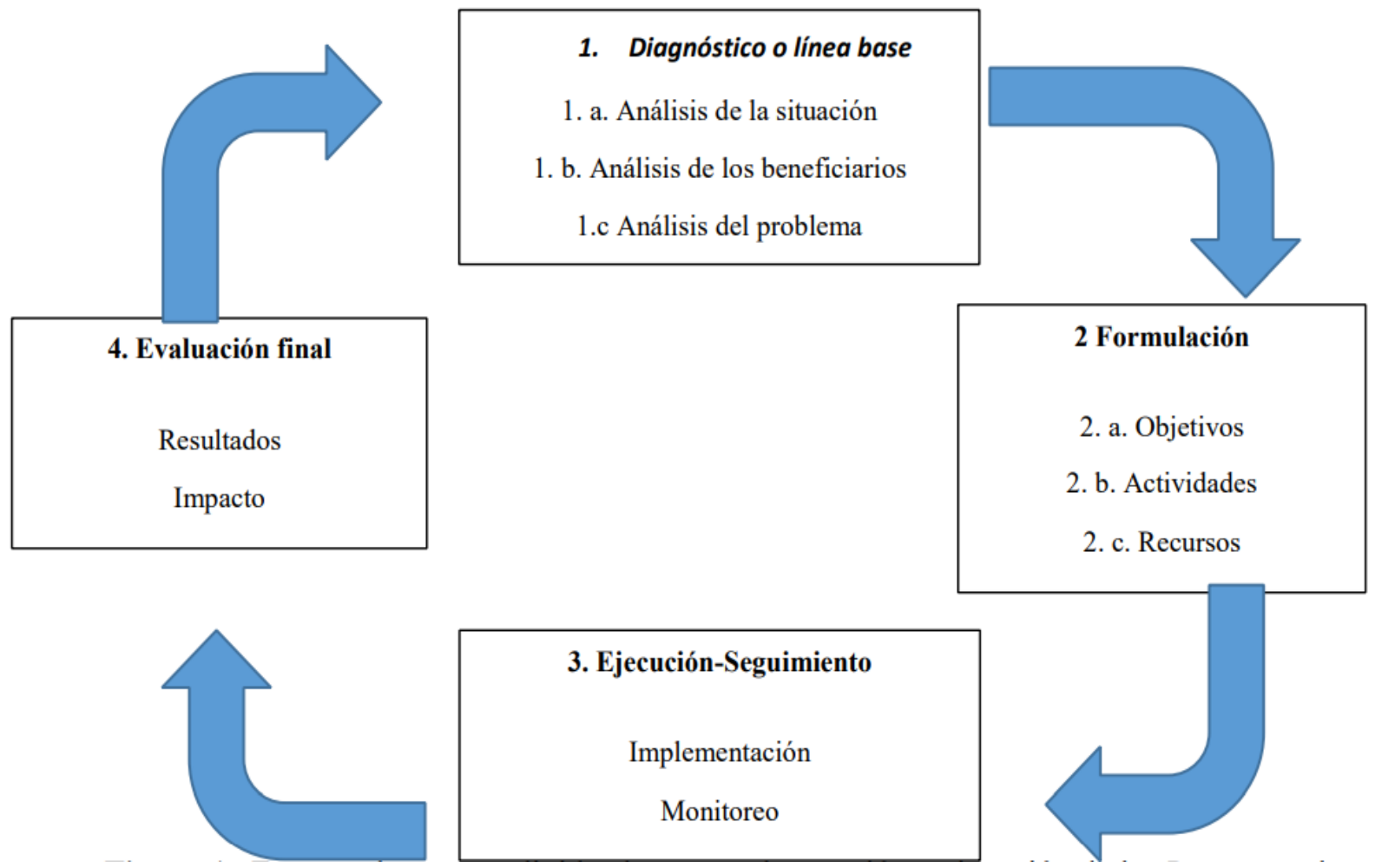

Fuente: Elaboración de los autores

- Diagnóstico o línea base, es la forma más segura de detectar un problema concreto, susceptible de mejorar mediante un conjunto de acciones organizadas. Un análisis profundo permite determinar las necesidades reales de los posibles grupos beneficiarios del proyecto. Los aspectos mínimos a tomar en cuenta serán:

○ Magnitud

- Grado de aceptabilidad

○ Viabilidad

- Potencialidad de conseguir el cambio deseado que involucre el análisis situacional o la realidad social

○ Análisis de beneficiarios directores, indirectos y los posibles resistentes, y

○ Análisis de problemas para determinar, las causas, efectos orígenes y consecuencias. 
- $\quad$ Formulación del proyecto, esta fase incorpora información generada en el diagnóstico. Primero identificamos el problema, después se desarrolla la forma de enfrentarse a él, haciendo visible la coherencia de los objetivos, resultados, acciones y tiempo disponible.

- $\quad$ Ejecución y seguimiento, la ejecución consiste en poner en marcha todas las actividades previstas en la formulación del proyecto. Es imprescindible un seguimiento que controle los avances y los desvíos; pues el seguimiento permite evaluar los resultados alcanzados y el impacto que han tenido sobre los beneficiarios. Para llevar a cabo la evaluación se usan indicadores e información generada en las distintas fases del proyecto. Algunas herramientas útiles son las entrevistas, los talleres de evaluación o encuestas de valoración.

- Evaluación final, implica la valoración sobre cómo se han ejecutado las acciones del proyecto, todos sus componentes, tareas, recursos, presupuesto, etc. El objetivo es garantizar la información permanente sobre esta ejecución que alimente así la toma de decisiones respecto a la eficiencia de los recursos previstos y la efectividad de las actividades desarrolladas. El diseño de esta evaluación también debe realizarse durante la planificación del proyecto evaluando indicadores de medición que permitan la recolección, ordenamiento, análisis e interpretación de información. En esta etapa es preciso explicitar y demostrar el logro de los resultados, al mismo tiempo, permitió diferenciar etapas proyecto y las consecuencias del proyecto.

\section{BENEFICIARIOS DE LOS PROGRAMAS Y PROYECTOS DE VINCULACIÓN DE LA}

\section{UNEMI 2012-2015}

Desde el 2011 en la UNEMI se han consolidado 9 Programas de Vinculación con sus respectivos proyectos (Tabla 02).

\begin{tabular}{cccc} 
Año & $\begin{array}{c}\text { Tabla 02. Número de proyectos y beneficiarios directos por año } \\
\text { N. de proyectos }\end{array}$ & $\begin{array}{c}\text { N. de beneficiarios directos } \\
\text { N. de beneficiarios } \\
\text { indirectos }\end{array}$ \\
\hline 2017 & 26 & 9533 & 35.288 \\
2016 & 29 & 8667 & 32.080 \\
2015 & 30 & 7879 & 29.164 \\
2014 & 45 & 7163 & 26.513 \\
2013 & 5 & 9467 & \\
2012 & 109 & 664 & \\
\hline
\end{tabular}

Fuente: Departamento de Extensión Universitaria - UNEMI, 2015

Los programas y proyectos de vinculación ejecutados por la UNEMI afectaron cuatro áreas establecidas por el CEAACES: Salud, Educación, Ingeniería y administración, áreas propiamente de las 
Facultades Académicas de la Universidad Estatal de Milagro siendo los beneficiarios principalmente de la zona 5 de la provincia del Guayas con un incremento aproximado del $10 \%$. 


\section{REFERENCIAS}

Arango Juan Carlos, Universidad de Medellín, Facultad de Ciencias Económicas y Administrativas, "Metodología de la investigación".

Bonilla P. Jorge W , "Diseño de proyectos Sociales con enfoque de marco lógico"

D`Este, P.; Martínez, E. y Molas - Gallart, J. (2009). Manual de Indicadores de Vinculación de la universidad con el entorno socioeconómico. Recuperado de: http://www.observatoriocts.org/files/Archivo\%20Documental/Documentos\%20de\%20proyect os/indicadores_de_vinculacion.pdf.

Ley Orgánica de Educación Superior, LOES (2010). Recuperado de: https://sangregorio.edu.ec/include/archivos/files/LOES_2.pdf

Mejía Carmona, A., Fernández Roque, T., Fernández Roque, J. A., \& Sandoval Lezama, J. (2020). Propuesta para mejorar la calidad en la práctica docente de los niveles medio y superior del IPN (México), a través de la formación del personal académico. South Florida Journal of Development,DOI: 10.46932/sfjdv2n2-023, 1414-1429.

REUVIC. (2012). Propuesta de Plan Nacional de Vinculación de la Educación con la Sociedad. $\begin{array}{llllll}\text { Recuperado el } & 28 & \text { de } & \text { junio } & 2016 & \text { de: }\end{array}$ http://www.reuvic.ec/reuvic/index.php?option=com_content\&view=article\&id=66:propuestad e-plannacional-de-vinculacion-de-la-educacion-con-la-sociedad\&catid=1:latestnews\&Itemid=16.

Secretaría Nacional de Planificación y Desarrollo. Zona de Planificación 5-Litoral. Recuperado de: http://www.planificacion.gob.ec/category/zona-de-planificacion-5-litoral/?cat=52.

Torres Hernández, Z., \& Torres Martínez, H. (2014). Planeación y control una visión integral de la administración. México: Grupo Editorial Patria.

USAID, "Guía de orientación No-2 Identificación, Formulación y Evaluación Social de Proyectos de inversión pública a nivel de perfil" 